\title{
Spectra and fine spectra of certain lower triangular double-band matrices as operators on $c_{0}$
}

Saad R El-Shabrawy*

\section{"Correspondence:}

srshabrawy@yahoo.com Mathematics Department, Faculty of Science, Damietta University, New Damietta, Egypt

\begin{abstract}
In this paper we determine the fine spectrum of the generalized difference operator $\Delta_{a, b}$ defined by a lower triangular double-band matrix over the sequence space $c_{0}$. The class of the operator $\Delta_{a, b}$ contains as special cases many operators that have been studied recently in the literature. Illustrative examples showing the advantage of the present results are also given.
\end{abstract}

MSC: Primary 47A10; secondary 47B37

Keywords: spectrum of an operator; infinite lower triangular matrices; sequence spaces

\section{Introduction}

Several authors have studied the spectrum and fine spectrum of linear operators defined by lower and upper triangular matrices over some sequence spaces [1-21].

Let $X$ be a Banach space. By $R(T), T^{*}, X^{*}, B(X), \sigma(T, X), \sigma_{p}(T, X), \sigma_{r}(T, X)$ and $\sigma_{c}(T, X)$, we denote the range of $T$, the adjoint operator of $T$, the space of all continuous linear functionals on $X$, the space of all bounded linear operators on $X$ into itself, the spectrum of $T$ on $X$, the point spectrum of $T$ on $X$, the residual spectrum of $T$ on $X$ and the continuous spectrum of $T$ on $X$, respectively. We shall write $c$ and $c_{0}$ for the spaces of all convergent and null sequences, respectively. Also by $l_{1}$ we denote the space of all absolutely summable sequences.

We assume here some familiarity with basic concepts of spectral theory and we refer to Kreyszig [22, pp.370-372] for basic definitions such as spectrum, point spectrum, residual spectrum, and continuous spectrum of linear operators in normed spaces. Also, we refer to Goldberg [23, pp.58-71] for Goldberg's classification of spectra.

Now, let $\left(a_{k}\right)$ and $\left(b_{k}\right)$ be two convergent sequences of nonzero real numbers with

$$
\lim _{k \rightarrow \infty} a_{k}=a \text { and } \quad \lim _{k \rightarrow \infty} b_{k}=b \neq 0 .
$$

We consider the operator $\Delta_{a, b}: c_{0} \rightarrow c_{0}$, which is defined as follows:

$$
\Delta_{a, b} x=\Delta_{a, b}\left(x_{k}\right)=\left(a_{k} x_{k}+b_{k-1} x_{k-1}\right)_{k=0}^{\infty} \quad \text { with } x_{-1}=b_{-1}=0 .
$$

@ 2014 El-Shabrawy; licensee Springer. This is an Open Access article distributed under the terms of the Creative Commons Attribution License (http://creativecommons.org/licenses/by/2.0), which permits unrestricted use, distribution, and reproduction in any medium, provided the original work is properly cited. 
It is easy to verify that the operator $\Delta_{a, b}$ can be represented by a lower triangular doubleband matrix of the form

$$
\Delta_{a, b}=\left(\begin{array}{cccc}
a_{0} & 0 & 0 & \ldots \\
b_{0} & a_{1} & 0 & \ldots \\
0 & b_{1} & a_{2} & \ldots \\
\vdots & \vdots & \vdots & \ddots
\end{array}\right) .
$$

We begin by determining when a matrix $A$ induces a bounded linear operator from $c_{0}$ to itself.

Lemma 1.1 ( $c f$. [24, p.129]) The matrix $A=\left(a_{n k}\right)$ gives rise to a bounded linear operator $T \in B\left(c_{0}\right)$ from $c_{0}$ to itself if and only if

(1) the rows of $A$ are in $l_{1}$ and their $l_{1}$ norms are bounded,

(2) the columns of $A$ are in $c_{0}$.

The operator norm of $T$ is the supremum of the $l_{1}$ norms of the rows.

As a consequence of the above lemma, we have the following corollary for the bounded linearity of the operator $\Delta_{a, b}$ on the space $c_{0}$.

Corollary 1.1 The operator $\Delta_{a, b}: c_{0} \longrightarrow c_{0}$ is a bounded linear operator with the norm $\left\|\Delta_{a, b}\right\|_{c_{0}}=\sup _{k}\left(\left|a_{k}\right|+\left|b_{k-1}\right|\right)$.

The rest of the paper is organized as follows. In Section 2, we analyze the spectrum of the operator $\Delta_{a, b}$ on the sequence space $c_{0}$. In Section 3 we give some illustrative examples. Finally, Section 4 concludes with remarks and some special cases.

\section{Fine spectrum of the operator $\Delta_{a, b}$ on $c_{0}$}

In this section we examine the spectrum, the point spectrum, the residual spectrum and the continuous spectrum of the operator $\Delta_{a, b}$ on the sequence space $c_{0}$.

Theorem 2.1 Let $D=\{\lambda \in \mathbb{C}:|\lambda-a| \leq|b|\}$ and $E=\left\{a_{k}: k \in \mathbb{N},\left|a_{k}-a\right|>|b|\right\}$. Then $\sigma\left(\Delta_{a, b}, c_{0}\right)=D \cup E$.

Proof First, we prove that $\left(\Delta_{a, b}-\lambda I\right)^{-1}$ exists and is in $B\left(c_{0}\right)$ for $\lambda \notin D \cup E$ and then the operator $\Delta_{a, b}-\lambda I$ is not invertible for $\lambda \in D \cup E$.

Let $\lambda \notin D \cup E$. Then $|\lambda-a|>|b|$ and $\lambda \neq a_{k}$, for all $k \in \mathbb{N}$. So, $\left(\Delta_{a, b}-\lambda I\right)$ is triangle and hence $\left(\Delta_{a, b}-\lambda I\right)^{-1}$ exists. We can calculate that

$$
\left(\Delta_{a, b}-\lambda I\right)^{-1}=\left(s_{k j}\right)=\left(\begin{array}{cccc}
\frac{1}{\left(a_{0}-\lambda\right)} & 0 & 0 & \ldots \\
\frac{-b_{0}}{\left(a_{0}-\lambda\right)\left(a_{1}-\lambda\right)} & \frac{1}{\left(a_{1}-\lambda\right)} & 0 & \ldots \\
\frac{b_{0} b_{1}}{\left(a_{0}-\lambda\right)\left(a_{1}-\lambda\right)\left(a_{2}-\lambda\right)} & \frac{-b_{1}}{\left(a_{1}-\lambda\right)\left(a_{2}-\lambda\right)} & \frac{1}{\left(a_{2}-\lambda\right)} & \ldots \\
\vdots & \vdots & \vdots & \ddots
\end{array}\right) .
$$

Now, for each $k \in \mathbb{N}$, the series $S_{k}=\sum_{j}\left|s_{k j}\right|$ is convergent since it is finite. Next, we prove that $\sup _{k} S_{k}$ is finite. 
Since $\lim _{k \rightarrow \infty}\left|\frac{b_{k}}{a_{k}-\lambda}\right|=\left|\frac{b}{a-\lambda}\right|<1$, then there exist $k_{0} \in \mathbb{N}$ and $q_{0}<1$ such that $\left|\frac{b_{k}}{a_{k}-\lambda}\right|<q_{0}$, for all $k \geq k_{0}$. Then, for each $k>k_{0}$,

$$
\begin{aligned}
S_{k}= & \frac{1}{\left|a_{k}-\lambda\right|}\left[1+\frac{\left|b_{k-1}\right|}{\left|a_{k-1}-\lambda\right|}+\frac{\left|b_{k-1}\right|\left|b_{k-2}\right|}{\left|a_{k-1}-\lambda\right|\left|a_{k-2}-\lambda\right|}\right. \\
& +\cdots+\frac{\left|b_{k-1}\right|\left|b_{k-2}\right| \cdots\left|b_{k_{0}}\right|}{\left|a_{k-1}-\lambda\right|\left|a_{k-2}-\lambda\right| \cdots\left|a_{k_{0}}-\lambda\right|} \\
& \left.+\cdots+\frac{\left|b_{k-1}\right|\left|b_{k-2}\right| \cdots\left|b_{k_{0}+1}\right|\left|b_{k_{0}}\right| \cdots\left|b_{0}\right|}{\left|a_{k-1}-\lambda\right|\left|a_{k-2}-\lambda\right| \cdots\left|a_{k_{0}+1}-\lambda\right|\left|a_{k_{0}}-\lambda\right| \cdots\left|a_{0}-\lambda\right|}\right] \\
\leq & \frac{1}{\left|a_{k}-\lambda\right|}\left[1+q_{0}+q_{0}^{2}+\cdots+q_{0}^{k-k_{0}}+q_{0}^{k-k_{0}} \frac{\left|b_{k_{0}-1}\right|}{\left|a_{k_{0}-1}-\lambda\right|}+q_{0}^{k-k_{0}} \frac{\left|b_{k_{0}-1}\right|\left|b_{k_{0}-2}\right|}{\left|a_{k_{0}-1}-\lambda\right|\left|a_{k_{0}-2}-\lambda\right|}\right. \\
& \left.+\cdots+q_{0}^{k-k_{0}} \frac{\left|b_{k_{0}-1}\right|\left|b_{k_{0}-2}\right| \cdots\left|b_{0}\right|}{\left|a_{k_{0}-1}-\lambda\right|\left|a_{k_{0}-2}-\lambda\right| \cdots\left|a_{0}-\lambda\right|}\right] .
\end{aligned}
$$

Therefore

$$
S_{k} \leq \frac{1}{\left|a_{k}-\lambda\right|}\left[1+q_{0}+q_{0}^{2}+\cdots+q_{0}^{k-k_{0}} m_{k_{0}}\right]
$$

where

$$
m_{k_{0}}=1+\frac{\left|b_{k_{0}-1}\right|}{\left|a_{k_{0}-1}-\lambda\right|}+\frac{\left|b_{k_{0}-1}\right|\left|b_{k_{0}-2}\right|}{\left|a_{k_{0}-1}-\lambda\right|\left|a_{k_{0}-2}-\lambda\right|}+\cdots+\frac{\left|b_{k_{0}-1}\right|\left|b_{k_{0}-2}\right| \cdots\left|b_{0}\right|}{\left|a_{k_{0}-1}-\lambda\right|\left|a_{k_{0}-2}-\lambda\right| \cdots\left|a_{0}-\lambda\right|} .
$$

Then $m_{k_{0}}>1$ and so

$$
S_{k} \leq \frac{m_{k_{0}}}{\left|a_{k}-\lambda\right|}\left[1+q_{0}+q_{0}^{2}+\cdots+q_{0}^{k-k_{0}}\right]
$$

But there exist $k_{1} \in \mathbb{N}$ and a real number $q_{1}<\frac{1}{|b|}$ such that $\frac{1}{\left|a_{k}-\lambda\right|}<q_{1}$, for all $k \geq k_{1}$. Then

$$
S_{k} \leq \frac{q_{1} m_{k_{0}}}{1-q_{0}}
$$

for all $k>\max \left\{k_{0}, k_{1}\right\}$. Thus $\sup _{k} S_{k}<\infty$.

Also, it is easy to see that $\lim _{k \rightarrow \infty}\left|s_{k j}\right|=0$, for all $j \in \mathbb{N}$, since

$$
\lim _{k \rightarrow \infty}\left|\frac{s_{k+1, j}}{s_{k, j}}\right|=\lim _{k \rightarrow \infty}\left|\frac{b_{k}}{a_{k+1}-\lambda}\right|=\left|\frac{b}{a-\lambda}\right|<1 .
$$

So, the sequence $\left(s_{0 j}, s_{1 j}, s_{2 j}, \ldots\right)$ converges to zero, for each $j \in \mathbb{N}$. This shows that the columns of $\left(\Delta_{a, b}-\lambda I\right)^{-1}$ are in $c_{0}$. Then, from Lemma 1.1, $\left(\Delta_{a, b}-\lambda I\right)^{-1} \in B\left(c_{0}\right)$ and so, $\lambda \notin \sigma\left(\Delta_{a, b}, c_{0}\right)$. Thus $\sigma\left(\Delta_{a, b}, c_{0}\right) \subseteq D \cup E$.

Conversely, suppose that $\lambda \notin \sigma\left(\Delta_{a, b}, c_{0}\right)$. Then $\left(\Delta_{a, b}-\lambda I\right)^{-1} \in B\left(c_{0}\right)$. Since the $\left(\Delta_{a, b}-\right.$ $\lambda I)^{-1}$ transform of the unite sequence $e_{0}=(1,0,0, \ldots)$ is in $c_{0}$, we have $\lim _{k \rightarrow \infty}\left|\frac{b_{k}}{a_{k+1}-\lambda}\right|=$ $\left|\frac{b}{a-\lambda}\right| \leq 1$ and $\lambda \neq a_{k}$, for all $k \in \mathbb{N}$. Then $\{\lambda \in \mathbb{C}:|\lambda-a|<|b|\} \subseteq \sigma\left(\Delta_{a, b}, c_{0}\right)$ and $\left\{a_{k}: k \in\right.$ $\mathbb{N}\} \subseteq \sigma\left(\Delta_{a, b}, c_{0}\right)$. But $\sigma\left(\Delta_{a, b}, c_{0}\right)$ is a compact set, and so it is closed. Then $D=\{\lambda \in \mathbb{C}$ : $|\lambda-a| \leq|b|\} \subseteq \sigma\left(\Delta_{a, b}, c_{0}\right)$ and $E=\left\{a_{k}: k \in \mathbb{N},\left|a_{k}-a\right|>|b|\right\} \subseteq \sigma\left(\Delta_{a, b}, c_{0}\right)$. This completes the proof. 
Theorem $2.2 \sigma_{p}\left(\Delta_{a, b}, c_{0}\right)=E \cup K$, where

$$
K=\left\{a_{j}: j \in \mathbb{N},\left|a_{j}-a\right|=|b|, \prod_{i=m}^{\infty} \frac{b_{i-1}}{a_{j}-a_{i}} \text { diverges to zero for some } m \in \mathbb{N}\right\} .
$$

Proof Suppose $\Delta_{a, b} x=\lambda x$ for any $x \in c_{0}$. Then we obtain

$$
\left(a_{0}-\lambda\right) x_{0}=0 \quad \text { and } \quad b_{k} x_{k}+\left(a_{k+1}-\lambda\right) x_{k+1}=0, \quad \text { for all } k \in \mathbb{N} .
$$

If the sequence $\left(a_{k}\right)$ is constant, then we can easily see that $x=\theta$ and so, $\sigma_{p}\left(\Delta_{a, b}, c_{0}\right)=\emptyset$ and the result follows immediately. Now, if the sequence $\left(a_{k}\right)$ is not constant, then for all $\lambda \notin\left\{a_{k}: k \in \mathbb{N}\right\}$, we have $x_{k}=0$ for all $k \in \mathbb{N}$. So, $\lambda \notin \sigma_{p}\left(\Delta_{a, b}, c_{0}\right)$. Also, we can easily prove that $a \notin \sigma_{p}\left(\Delta_{a, b}, c_{0}\right)$. Thus $\sigma_{p}\left(\Delta_{a, b}, c_{0}\right) \subseteq\left\{a_{k}: k \in \mathbb{N}\right\} \backslash\{a\}$. Now, we will prove that

$$
\lambda \in \sigma_{p}\left(\Delta_{a, b}, c_{0}\right) \quad \text { if and only if } \quad \lambda \in E \cup K .
$$

If $\lambda \in \sigma_{p}\left(\Delta_{a, b}, c_{0}\right)$, then $\lambda=a_{j} \neq a$ for some $j \in \mathbb{N}$ and there exists $x \in c_{0}, x \neq \theta$ such that $\Delta_{a, b} x=a_{j} x$. Then

$$
\lim _{k \rightarrow \infty}\left|\frac{x_{k+1}}{x_{k}}\right|=\left|\frac{b}{a-a_{j}}\right| \leq 1
$$

Then $\lambda=a_{j} \in E$ or $\left|a_{j}-a\right|=|b|$. In the case when $\left|a_{j}-a\right|=|b|$, we have

$$
x_{k}=\frac{b_{k-1} b_{k-2} \cdots b_{m-1}}{\left(a_{j}-a_{k}\right)\left(a_{j}-a_{k-1}\right) \cdots\left(a_{j}-a_{m}\right)} x_{m-1}=x_{m-1} \prod_{i=m}^{k} \frac{b_{i-1}}{a_{j}-a_{i}}, \quad k \geq m .
$$

Then $\prod_{i=m}^{\infty} \frac{b_{i-1}}{a_{j}-a_{i}}$ diverges to 0 , since $x \in c_{0}$. Therefore $\lambda \in E \cup K$. Thus $\sigma_{p}\left(\Delta_{a, b}, c_{0}\right) \subseteq E \cup K$. Conversely, let $\lambda \in E \cup K$. If $\lambda \in E$, then there exists $i \in \mathbb{N}$ such that $\lambda=a_{i} \neq a$ and so we can take $x \neq \theta$ such that $\Delta_{a, b} x=a_{i} x$ and

$$
\lim _{k \rightarrow \infty}\left|\frac{x_{k+1}}{x_{k}}\right|=\left|\frac{b}{a-a_{i}}\right|<1
$$

that is, $x \in c_{0}$. Also, if $\lambda \in K$, then there exists $j \in \mathbb{N}$ such that $\lambda=a_{j} \neq a$ and $\left|a_{j}-a\right|=|b|$, $\prod_{i=m}^{\infty} \frac{b_{i-1}}{a_{j}-a_{i}}$ diverges to 0 , for some $m \in \mathbb{N}$. Then we can take $x \in c_{0}, x \neq \theta$ such that $\Delta_{a, b} x=$ $a_{j} x$. Thus $E \cup K \subseteq \sigma_{p}\left(\Delta_{a, b}, c_{0}\right)$. This completes the proof.

Theorem 2.3 $\sigma_{p}\left(\Delta_{a, b}^{*}, c_{0}^{*}\right)=\{\lambda \in \mathbb{C}:|\lambda-a|<|b|\} \cup E \cup H$, where

$$
H=\left\{\lambda \in \mathbb{C}:|\lambda-a|=|b|, \sum_{k=0}^{\infty}\left|\prod_{i=0}^{k} \frac{\lambda-a_{i}}{b_{i}}\right|<\infty\right\} .
$$


Proof Suppose that $\Delta_{a, b}^{*} f=\lambda f$ for $f=\left(f_{0}, f_{1}, f_{2}, \ldots\right) \neq \theta$ in $c_{0}^{*} \cong l_{1}$. Then, by solving the system of linear equations

$$
\begin{aligned}
& a_{0} f_{0}+b_{0} f_{1}=\lambda f_{0}, \\
& a_{1} f_{1}+b_{1} f_{2}=\lambda f_{1}, \\
& a_{2} f_{2}+b_{2} f_{3}=\lambda f_{2},
\end{aligned}
$$

$$
\vdots
$$

we obtain

$$
f_{k+1}=\frac{\lambda-a_{k}}{b_{k}} f_{k}, \quad k \in \mathbb{N}
$$

Then we must take $f_{0} \neq 0$ since otherwise we would have $f=\theta$. It is clear that for all $k \in \mathbb{N}$, the vector $f=\left(f_{0}, f_{1}, \ldots, f_{k}, 0,0, \ldots\right)$ is an eigenvector of the operator $\Delta_{a, b}^{*}$ corresponding to the eigenvalue $\lambda=a_{k}$, where $f_{0} \neq 0$ and $f_{n}=\frac{\lambda-a_{n-1}}{b_{n-1}} f_{n-1}$, for all $n=1,2,3, \ldots, k$. Then $\left\{a_{k}: k \in\right.$ $\mathbb{N}\} \subseteq \sigma_{p}\left(\Delta_{a, b}^{*}, c_{0}^{*}\right)$. Also, if $\lambda \neq a_{k}$ for all $k \in \mathbb{N}$, then $f_{k} \neq 0$, for all $k \geq 1$ and $\sum_{k=0}^{\infty}\left|f_{k}\right|<\infty$ if $\lim _{k \rightarrow \infty}\left|\frac{f_{k+1}}{f_{k}}\right|=\left|\frac{\lambda-a}{b}\right|<1$. Also, if $|\lambda-a|=|b|$, we can easily see that

$$
\left|f_{k}\right|=\left|\frac{\left(\lambda-a_{0}\right)\left(\lambda-a_{1}\right) \cdots\left(\lambda-a_{k-1}\right)}{b_{0} b_{1} \cdots b_{k-1}}\right|\left|f_{0}\right|=\left|f_{0}\right| \prod_{i=0}^{k-1}\left|\frac{\lambda-a_{i}}{b_{i}}\right|, \quad \text { for all } k \geq 1,
$$

and so $\sum_{k=0}^{\infty}\left|f_{k}\right|<\infty$ if $\sum_{k=0}^{\infty}\left|\prod_{i=0}^{k} \frac{\lambda-a_{i}}{b_{i}}\right|<\infty$. This implies that $H \subseteq \sigma_{p}\left(\Delta_{a, b}^{*}, c_{0}^{*}\right)$. Thus

$$
\{\lambda \in \mathbb{C}:|\lambda-a|<|b|\} \cup E \cup H \subseteq \sigma_{p}\left(\Delta_{a, b}^{*}, c_{0}^{*}\right) .
$$

The second inclusion can be proved analogously.

The following lemma is required in the proof of the next theorem.

Lemma 2.1 [23, p.59] $T$ has a dense range if and only if $T^{*}$ is one to one.

Theorem 2.4 $\sigma_{r}\left(\Delta_{a, b}, c_{0}\right)=\sigma_{p}\left(\Delta_{a, b}^{*}, c_{0}^{*}\right) \backslash \sigma_{p}\left(\Delta_{a, b}, c_{0}\right)$.

Proof For $\lambda \in \sigma_{p}\left(\Delta_{a, b}^{*}, c_{0}^{*}\right) \backslash \sigma_{p}\left(\Delta_{a, b}, c_{0}\right)$, the operator $\Delta_{a, b}-\lambda I$ is one to one and hence has an inverse. But $\Delta_{a, b}^{*}-\lambda I$ is not one to one. Now, Lemma 2.1 yields the fact that the range of the operator $\Delta_{a, b}-\lambda I$ is not dense in $c_{0}$. This implies that $\lambda \in \sigma_{r}\left(\Delta_{a, b}, c_{0}\right)$.

Theorem $2.5 \sigma_{r}\left(\Delta_{a, b}, c_{0}\right)=\{\lambda \in \mathbb{C}:|\lambda-a|<|b|\} \cup(H \backslash K)$.

Proof The proof follows immediately from Theorems 2.2, 2.3, and 2.4.

Theorem 2.6 $\sigma_{c}\left(\Delta_{a, b}, c_{0}\right)=\{\lambda \in \mathbb{C}:|\lambda-a|=|b|\} \backslash H$.

Proof Since $\sigma\left(\Delta_{a, b}, c_{0}\right)$ is the disjoint union of the parts $\sigma_{p}\left(\Delta_{a, b}, c_{0}\right), \sigma_{r}\left(\Delta_{a, b}, c_{0}\right)$ and $\sigma_{c}\left(\Delta_{a, b}, c_{0}\right)$ we must have $\sigma_{c}\left(\Delta_{a, b}, c_{0}\right)=\{\lambda \in \mathbb{C}:|\lambda-a|=|b|\} \backslash H$.

Also, we have the following result. 
Theorem $2.7 \sigma_{c}\left(\Delta_{a, b}, c_{0}\right)=\sigma\left(\Delta_{a, b}, c_{0}\right) \backslash \sigma_{p}\left(\Delta_{a, b}^{*}, c_{0}^{*}\right)$.

Proof The proof is obvious and so is omitted.

With respect to Goldberg's classification of the spectrum of an operator (see [23, pp.5871]), the spectrum is partitioned into nine states, which are $I_{1}, I_{2}, I_{3}, I I_{1}, I I_{2}, I I_{3}, I I I_{1}, I I I_{2}$, and $I I I_{3}$. For the operator $\Delta_{a, b}: c_{0} \rightarrow c_{0}$, we have

$$
I_{3} \sigma\left(\Delta_{a, b}, c_{0}\right)=I I_{3} \sigma\left(\Delta_{a, b}, c_{0}\right)=\emptyset,
$$

since $\sigma_{p}\left(\Delta_{a, b}, c_{0}\right) \subseteq \sigma_{p}\left(\Delta_{a, b}^{*}, c_{0}^{*}\right)$. Also, $I_{2} \sigma\left(\Delta_{a, b}, c_{0}\right)=\emptyset$, by the closed graph theorem. Thus we have to discuss the states $I I_{2}, I I I_{1}, I I I_{2}$, and $I I I_{3}$.

Theorem $2.8 \lambda \in \sigma_{p}\left(\Delta_{a, b}, c_{0}\right)$ if and only if $\lambda \in I I_{3} \sigma\left(\Delta_{a, b}, c_{0}\right)$.

Proof The proof is obvious and so is omitted.

Theorem $2.9 \lambda \in \sigma_{c}\left(\Delta_{a, b}, c_{0}\right)$ if and only if $\lambda \in I I_{2} \sigma\left(\Delta_{a, b}, c_{0}\right)$.

Proof Let $\lambda \in \sigma_{c}\left(\Delta_{a, b}, c_{0}\right)$. By Theorem 2.7, $\Delta_{a, b}^{*}-\lambda I$ is one to one. By Lemma 2.1, $\Delta_{a, b}-$ $\lambda I$ has dense range. Additionally, $\lambda \notin \sigma_{p}\left(\Delta_{a, b}, c_{0}\right)$ implies that the operator $\Delta_{a, b}-\lambda I$ has inverse. Therefore, $\lambda \in I I_{2} \sigma\left(\Delta_{a, b}, c_{0}\right)$ or $\lambda \in I_{2} \sigma\left(\Delta_{a, b}, c_{0}\right)$. But $I_{2} \sigma\left(\Delta_{a, b}, c_{0}\right)=\emptyset$. Thus $\lambda \in$ $I I_{2} \sigma\left(\Delta_{a, b}, c_{0}\right)$.

Theorem 2.10 $\lambda \in \sigma_{r}\left(\Delta_{a, b}, c_{0}\right)$ if and only if $\lambda \in I I I_{1} \sigma\left(\Delta_{a, b}, c_{0}\right) \cup I I I_{2} \sigma\left(\Delta_{a, b}, c_{0}\right)$.

Proof Let $\lambda \in \sigma_{r}\left(\Delta_{a, b}, c_{0}\right)$. By Theorem 2.4, $\Delta_{a, b}^{*}-\lambda I$ is not one to one. By Lemma 2.1, $\Delta_{a, b}-\lambda I$ has not a dense range. Additionally, $\lambda \notin \sigma_{p}\left(\Delta_{a, b}, c_{0}\right)$ implies that the operator $\Delta_{a, b}-\lambda I$ has inverse. Therefore, $\lambda \in I I I_{1} \sigma\left(\Delta_{a, b}, c_{0}\right) \cup I I I_{2} \sigma\left(\Delta_{a, b}, c_{0}\right)$.

\section{Illustrative examples}

In this section we provide some illustrative examples in support of our new results.

Example 3.1 Consider the sequences $\left(a_{k}\right)$ and $\left(b_{k}\right)$ defined by the following recurrence relations:

$$
\begin{array}{ll}
a_{0}=\sqrt{2}, & a_{k+1}=\sqrt{2+a_{k}}, \\
b_{0}=\sqrt{2}, & b_{k+1}=\sqrt{2 b_{k}},
\end{array}
$$

for all $k \in \mathbb{N}$. Then $\left(a_{k}\right)$ and $\left(b_{k}\right)$ are monotonically increasing sequences and $\lim _{k \rightarrow \infty} a_{k}=$ $a=2$ and $\lim _{k \rightarrow \infty} b_{k}=b=2$. Also, $a_{k} \geq b_{k}$ for all $k \in \mathbb{N}$. Thus, for all $\lambda \in \mathbb{C}$ with $|\lambda-2|=2$, one can prove that $\left|\frac{\lambda-a_{k}}{b_{k}}\right| \geq 1$ for all $k \in \mathbb{N}$. This implies that $H=\emptyset$. Also, we can prove that $E=K=\emptyset$. Using Theorems 2.1, 2.2, 2.5, and 2.6, we have

$$
\begin{aligned}
& \sigma\left(\Delta_{a, b}, c_{0}\right)=\{\lambda \in \mathbb{C}:|\lambda-2| \leq 2\}, \\
& \sigma_{p}\left(\Delta_{a, b}, c_{0}\right)=\emptyset
\end{aligned}
$$




$$
\begin{aligned}
& \sigma_{r}\left(\Delta_{a, b}, c_{0}\right)=\{\lambda \in \mathbb{C}:|\lambda-2|<2\}, \\
& \sigma_{c}\left(\Delta_{a, b}, c_{0}\right)=\{\lambda \in \mathbb{C}:|\lambda-2|=2\} .
\end{aligned}
$$

Example 3.2 Let $a_{k}=\frac{k+1}{k+2}$ and $b_{k}=\frac{k+1}{k+3}$ for all $k \in \mathbb{N}$. Then $\lim _{k \rightarrow \infty} a_{k}=a=1$ and $\lim _{k \rightarrow \infty} b_{k}=b=1$. Similarly, as in Example 3.1, we can prove that $E=K=H=\emptyset$ and so

$$
\begin{aligned}
& \sigma\left(\Delta_{a, b}, c_{0}\right)=\{\lambda \in \mathbb{C}:|\lambda-1| \leq 1\}, \\
& \sigma_{p}\left(\Delta_{a, b}, c_{0}\right)=\emptyset, \\
& \sigma_{r}\left(\Delta_{a, b}, c_{0}\right)=\{\lambda \in \mathbb{C}:|\lambda-1|<1\}, \\
& \sigma_{c}\left(\Delta_{a, b}, c_{0}\right)=\{\lambda \in \mathbb{C}:|\lambda-1|=1\} .
\end{aligned}
$$

Example 3.3 Consider the sequences $\left(a_{k}\right)$ and $\left(b_{k}\right)$ defined by the following recurrence relations:

$$
\begin{array}{ll}
a_{0}=4, & a_{1}=2, \quad a_{k}=1 \quad \text { for } k \geq 2, \\
b_{0}=1, \quad b_{1}=1, & b_{k}=\left(\frac{k+1}{k}\right)^{2} \quad \text { for } k \geq 2 .
\end{array}
$$

Therefore, $\lim _{k \rightarrow \infty} a_{k}=a=1$ and $\lim _{k \rightarrow \infty} b_{k}=b=1$. Then $E=\{4\}, K=\emptyset$ and $H=\{\lambda \in \mathbb{C}$ : $|\lambda-1|=1\}$, and so

$$
\begin{aligned}
& \sigma\left(\Delta_{a, b}, c_{0}\right)=\{\lambda \in \mathbb{C}:|\lambda-1| \leq 1\} \cup\{4\}, \\
& \sigma_{p}\left(\Delta_{a, b}, c_{0}\right)=\{4\}, \\
& \sigma_{r}\left(\Delta_{a, b}, c_{0}\right)=\{\lambda \in \mathbb{C}:|\lambda-1| \leq 1\}, \\
& \sigma_{c}\left(\Delta_{a, b}, c_{0}\right)=\emptyset .
\end{aligned}
$$

Remark 3.1 From the above examples, we note that the spectrum of the operator $\Delta_{a, b}$ on the space $c_{0}$ may include also a finite number of points outside a region enclosed by a circle. Also, we may have $\sigma_{p}\left(\Delta_{a, b}, c_{0}\right) \neq \emptyset$.

Example 3.4 Let the sequences $\left(a_{k}\right)$ and $\left(b_{k}\right)$ be taken such that $a_{k}=-b_{k}=\frac{(k+2)^{2}}{(k+2)^{2}+(k+3)^{2}}$, $k \in \mathbb{N}$. Then we can prove that $E=K=H=\emptyset$ and so we have

$$
\begin{aligned}
& \sigma\left(\Delta_{a, b}, c_{0}\right)=\left\{\lambda \in \mathbb{C}:\left|\lambda-\frac{1}{2}\right| \leq \frac{1}{2}\right\}, \\
& \sigma_{p}\left(\Delta_{a, b}, c_{0}\right)=\emptyset, \\
& \sigma_{r}\left(\Delta_{a, b}, c_{0}\right)=\left\{\lambda \in \mathbb{C}:\left|\lambda-\frac{1}{2}\right|<\frac{1}{2}\right\}, \\
& \sigma_{c}\left(\Delta_{a, b}, c_{0}\right)=\left\{\lambda \in \mathbb{C}:\left|\lambda-\frac{1}{2}\right|=\frac{1}{2}\right\} .
\end{aligned}
$$




\section{Remarks and some special cases}

In this section we are going to give some special cases of the operator $\Delta_{a, b}$ which has been studied recently. More precisely, we show that special conditions on the sequences $\left(a_{k}\right)$ and $\left(b_{k}\right)$ characterize certain special cases of the operator $\Delta_{a, b}$.

The difference operator $\Delta$ : If $a_{k}=1$ and $b_{k}=-1$ for all $k \in \mathbb{N}$, then the operator $\Delta_{a, b}$ reduces to the backward difference operator $\Delta$ (cf. [7]).

The generalized difference operator $B(r, s)$ : If $a_{k}=r$ and $b_{k}=s \neq 0$ for all $k \in \mathbb{N}$, then the operator $\Delta_{a, b}$ reduces to the operator $B(r, s)(c f$. [8]).

The generalized difference operator $\Delta_{v}$ : If $a_{k}=-b_{k}=v_{k}$ for all $k \in \mathbb{N}$, then the operator $\Delta_{a, b}$ reduces to the operator $\Delta_{v}(c f .[19])$.

The generalized difference operator $\Delta_{u v}$ : If $\left(a_{k}\right)$ is a sequence of positive real numbers such that $a_{k} \neq 0$ for all $k \in \mathbb{N}$ with $\lim _{k \rightarrow \infty} a_{k}=U \neq 0$ and $\left(b_{k}\right)$ is either constant or strictly decreasing sequence of positive real numbers with $\lim _{k \rightarrow \infty} b_{k}=V \neq 0$ and $\sup _{k} a_{k}<U+V$, then the operator $\Delta_{a, b}$ reduces to the operator $\Delta_{u v}(c f .[12])$.

Remark 4.1 If $\left(a_{k}\right)$ and $\left(b_{k}\right)$ are convergent sequences of nonzero real numbers such that

$$
\begin{aligned}
& \lim _{k \rightarrow \infty} a_{k}=a>0, \\
& \lim _{k \rightarrow \infty} b_{k}=b ; \quad|b|=a,
\end{aligned}
$$

and

$$
\sup _{k} a_{k} \leq a, \quad b_{k}^{2} \leq a_{k}^{2} \quad \text { for all } k \in \mathbb{N}
$$

then we can prove that $H=\emptyset$ and so we have:

$$
\begin{aligned}
& \sigma\left(\Delta_{a, b}, c_{0}\right)=\{\lambda \in \mathbb{C}:|\lambda-a| \leq a\} \cup\left\{a_{k}: k \in \mathbb{N},\left|a_{k}-a\right|>a\right\}, \\
& \sigma_{p}\left(\Delta_{a, b}, c_{0}\right)=\left\{a_{k}: k \in \mathbb{N},\left|a_{k}-a\right|>a\right\}, \\
& \sigma_{p}\left(\Delta_{a, b}^{*}, c_{0}^{*}\right)=\{\lambda \in \mathbb{C}:|\lambda-a|<a\} \cup\left\{a_{k}: k \in \mathbb{N},\left|a_{k}-a\right|>a\right\}, \\
& \sigma_{r}\left(\Delta_{a, b}, c_{0}\right)=\{\lambda \in \mathbb{C}:|\lambda-a|<a\}, \\
& \sigma_{c}\left(\Delta_{a, b}, c_{0}\right)=\{\lambda \in \mathbb{C}:|\lambda-a|=a\} .
\end{aligned}
$$

It is immediate that our new results cover a wider class of linear operators which are represented by infinite lower triangular double-band matrices on the sequence space $c_{0}$. For this reason, our study is more general and more comprehensive than the previous work. We note that our new results in this paper improve and generalize the results which have been stated in $[3,12]$.

Competing interests

The author declares that he has no competing interests.

\section{Acknowledgements}

I wish to express my thanks to Prof. Ali M Akhmedov, Baku State University, Faculty of Mechanics \& Mathematics, Baku, Azerbaijan, for his kind help, careful reading, and making useful comments on the earlier version of this paper. Also, I thank the editor and the anonymous referees for their careful reading and making some useful comments which improved the presentation of the paper. 


\section{References}

1. Akhmedov, AM, Başar, F: On the fine spectra of the difference operator $\Delta$ over the sequence space $I_{p}(1 \leq p<\infty)$. Demonstr. Math. 39(3), 585-595 (2006)

2. Akhmedov, AM, Başar, $\mathrm{F}$ : The fine spectra of the difference operator $\Delta$ over the sequence space $b v_{p}(1 \leq p<\infty)$. Acta Math. Sin. Engl. Ser. 23(10), 1757-1768 (2007)

3. Akhmedov, AM, El-Shabrawy, SR: On the spectrum of the generalized difference operator $\Delta_{a, b}$ over the sequence space $c_{0}$. Baku Univ. News J., Phys. Math. Sci. Ser. 4, 12-21 (2010)

4. Akhmedov, AM, El-Shabrawy, SR: On the fine spectrum of the operator $\Delta_{a, b}$ over the sequence space $c$. Comput. Math. Appl. 61, 2994-3002 (2011)

5. Akhmedov, AM, El-Shabrawy, SR: On the fine spectrum of the operator $\Delta_{V}$ over the sequence spaces $\mathrm{C}$ and $I_{p}$ $(1<p<\infty)$. Appl. Math. Inf. Sci. 5(3), 635-654 (2011)

6. Akhmedov, AM, El-Shabrawy, SR: Spectra and fine spectra of lower triangular double-band matrices as operators on Ip $(1<p<\infty)$. Math. Slovaca (accepted)

7. Altay, B, Başar, F: On the fine spectrum of the difference operator $\Delta$ on $c_{0}$ and C. Inf. Sci. 168, $217-224$ (2004)

8. Altay, B, Başar, F: On the fine spectrum of the generalized difference operator $B(r, s)$ over the sequence spaces $C_{0}$ and c. Int. J. Math. Math. Sci. 18, 3005-3013 (2005)

9. Başar, F: Summability Theory and Its Applications. Bentham Science Publishers, Istanbul (2012)

10. Bilgiç, $H$, Furkan, $H$ : On the fine spectrum of the generalized difference operator $B(r, s)$ over the sequence spaces $I_{p}$ and $b v_{p}(1<p<\infty)$. Nonlinear Anal. 68, 499-506 (2008)

11. El-Shabrawy, SR: On the fine spectrum of the generalized difference operator $\Delta_{a, b}$ over the sequence space $I_{p}$ $(1<p<\infty)$. Appl. Math. Inf. Sci. 6(1S), Special Issue, 111-118 (2012)

12. Fathi, J, Lashkaripour, R: On the fine spectra of the generalized difference operator $\Delta_{u v}$ over the sequence space $c_{0}$. J. Mahani Math. Res. Cent. 1(1), 1-12 (2012)

13. Furkan, $\mathrm{H}$, Bilgiç, $H$, Altay, B: On the fine spectrum of the operator $B(r, s, t)$ over $c_{0}$ and $c$. Comput. Math. Appl. 53 , 989-998 (2007)

14. Furkan, $H$, Bilgiç, $H$, Başar, $F$ : On the fine spectrum of the operator $B(r, s, t)$ over the sequence spaces $I_{p}$ and $b v_{p}$ $(1<p<\infty)$. Comput. Math. Appl. 60, 2141-2152 (2010)

15. Karaisa, $A$ : Fine spectra of upper triangular double-band matrices over the sequence space $I_{p}(1<p<\infty)$. Discrete Dyn. Nat. Soc. 2012, Article ID 381069 (2012)

16. Karaisa, A, Başar, F: Fine spectra of upper triangular triple-band matrices over the sequence space $I_{p}(0<p<\infty)$. Abstr. Appl. Anal. 2013, Article ID 342682 (2013)

17. Karakaya, V, Altun, M: Fine spectra of upper triangular double-band matrices. J. Comput. Appl. Math. 234, 1387-1394 (2010)

18. Panigrahi, BL, Srivastava, PD: Spectrum and fine spectrum of generalized second order difference operator $\Delta_{u v}^{2}$ on sequence space $c_{0}$. Thai J. Math. 9(1), 57-74 (2011)

19. Srivastava, PD, Kumar, $\mathrm{S}$ : On the fine spectrum of the generalized difference operator $\Delta_{V}$ over the sequence space $c_{0}$ Commun. Math. Anal. 6(1), 8-21 (2009)

20. Srivastava, PD, Kumar, S: Fine spectrum of generalized difference operator $\Delta_{V}$ over the sequence space $I_{1}$. Thai J. Math. 8(2), 221-233 (2010)

21. Tripathy, BC, Saikia, P: On the spectrum of the Cesàro operator $C_{1}$ on $\overline{b v_{0}} \cap /_{\infty}$. Math. Slovaca 63(3), 563-572 (2013)

22. Kreyszig, E: Introductory Functional Analysis with Applications. Wiley, New York (1978)

23. Goldberg, S: Unbounded Linear Operators: Theory and Applications. McGraw-Hill, New York (1966)

24. Wilansky, A: Summability Through Functional Analysis. North-Holland Mathematics Studies, vol. 85. North-Holland, Amsterdam (1984)

doi:10.1186/1029-242X-2014-241

Cite this article as: El-Shabrawy: Spectra and fine spectra of certain lower triangular double-band matrices as operators on $c_{0}$. Journal of Inequalities and Applications 2014 2014:241. 